\title{
An electrically tunable single-photon source triggered by a monolithically integrated quantum dot microlaser
}

Pierce Munnelly, ${ }^{\dagger}$ Tobias Heindel, ${ }^{\dagger}$ Alexander Thoma ${ }^{\dagger}$ Martin Kamp, $^{\ddagger}$ Sven Höfling, ${ }^{\ddagger}$ Christian Schneider, ${ }^{\ddagger}$ and Stephan Reitzenstein ${ }^{*, \dagger}$

$\dagger$ Institut für Festkörperphysik, Technische Universität Berlin, 10623 Berlin, Germany $\ddagger$ Technische Physik, Universität Würzburg, 97074 Würzburg, Germany

\School of Physics and Astronomy, University of St Andrews, St Andrews KY16 9SS, United Kingdom

E-mail: stephan.reitzenstein@physik.tu-berlin.de

\begin{abstract}
We report on a quantum dot micropillar-based single-photon source demonstrating tunable emission energy via an applied electric field and driven by an on-chip, whispering-gallery-mode microlaser. The cavity-enhanced single-photon source is monolithically integrated with an electrically driven, coherent excitation source. The device concept features low laser-threshold currents of a few tens of $\mu \mathrm{A}$, has a small footprint with a device area of $\sim 200 \mu \mathrm{m}^{2}$ and demonstrates high single-photon purity with $g^{(2)}(0)$ as low as $0.07 \pm 0.03$ under pulsed electrical excitation of the microlaser. The electric field applied along the growth direction of the single-photon emitter allows the emission to be tuned by up to $1.1 \mathrm{meV}$ via the quantum-confined Stark effect, bringing it into resonance with the fundamental mode of the surrounding micropillar resonator for enhanced emission via the Purcell effect.
\end{abstract}




\section{Keywords}

Semiconductor Quantum Dot, Whispering-Gallery-Mode Laser, Single-Photon Source, Photonics, Integrated Quantum Optics

Quantum dots (QDs) in the solid-state are well-suited for on-demand production of single photons, which themselves are of great interest for quantum information processing, communication and metrology. ${ }^{1,2}$ Two withstanding challenges for successful applications of QD-based single-photon sources (SPSs) are integration with other photonic elements and control over the emission properties.

Regarding integration, directional and enhanced luminescence from QDs has been successfully achieved by, for instance, coupling to waveguides, ${ }^{3}$ nano-antennae, ${ }^{4,5}$ nanowires, ${ }^{6}$ microlenses ${ }^{7}$ and micropillar cavities. ${ }^{8,9}$ In particular, the GaAs platform has proven to be appropriate in many ways for photonic integration. ${ }^{10}$ Furthermore, the requirement of indistinguishability necessary for quantum technologies relying on single-photon interference and entanglement distribution will best be met with resonant excitation schemes. ${ }^{11-13}$ In the interest of compactness and scalability, electrical carrier injection would be highly attractive - avoiding bulky external laser sources. However, simple electrically driven sources based on a pin-diode design do not allow for resonant excitation and suffer from charge noise introduced by excess carriers, both of which limit the indistinguishability of the emitted photons. A possible solution to this problem is the integration of an optical excitation source on the same chip, which is itself electrically triggered, spectrally narrow and allows for resonant excitation of the target single-photon emitter as we proposed and demonstrated in Ref. ${ }^{14}$

Regarding control of the QD emission energy, this has commonly been achieved in one of three ways: temperature tuning, ${ }^{8}$ which exploits the temperature dependence of the bandgap; piezo-electric strain tuning, ${ }^{15}$ which relies on the built-in polarization of the matrix material to modify the energy levels of the emitter via the quantum-confined Stark effect (QCSE); and by direct application of an electric field, ${ }^{16}$ which also modifies the emission energy via the QCSE. Temperature tuning is slow and not scalable for integrated systems 
of separate photonic elements which rely on a constant refractive index, such as waveguides; strain tuning has made great progress in recent years ${ }^{15,17}$ but requires either complicated processing steps or large piezo-actuators which again limit scalability. It seems the most desirable tuning mechanism would be able to tune the emitters with minimal fabrication complexity and in a localized way that does not interfere with other integrated elements. QCSE via an applied electric field is an ideal mechanism for this, showing large tuning ranges and tuning areas limited by the contact size. ${ }^{18-22}$

Monolithic integration of QDs with coherent, electrically controlled whispering-gallerymode (WGM) lasers has recently been pursued. ${ }^{23}$ This work represents a major advancement in this direction by bringing multiple aspects of desirable functionality, including integration and electrical control, together on a single chip as well as very pure single-photon emission.

\section{Methods}

The monolithically integrated microlaser and electrically tunable SPS consist of pin-doped QD-based micropillars grown by molecular beam epitaxy on an n-doped GaAs substrate. A single layer of InGaAs quantum dots (areal density $3 \times 10^{9} \mathrm{~cm}^{-2}$ ) embedded within a $\lambda$-cavity form the active material for WGM lasing in the larger of the two micropillars, henceforth referred to as the microlaser. The same QD layer provides the single-photon emitters in the smaller of the two micropillars, henceforth referred to as the SPS-micropillar; for fabrication details see Ref. ${ }^{23}$ The fundamental pillar mode is spectrally detuned from the QD ensemble to the red side of its maximum by $\sim 50 \mathrm{meV}$, which results in a spectral density of about one excitonic line per meV in the spectral vicinity of the cavity mode facilitating single QD studies. The device characterized here has an optimized number of distributed-Bragg reflector (DBR) pairs of 13 above and 26 below the cavity for the best compromise between brightness and Purcell enhancement in the SPS. ${ }^{24}$ The microlaser and SPS-micropillar are planarized in the transparent polymer benzocyclobutene $(\mathrm{BCB})$ to support the upper $\mathrm{p}$ - 
contacts for electrical control. The micropillars share the same n-contact on the back side, but have isolated p-contacts allowing for the individual control of the current injected into the microlaser or the bias-voltage applied to the SPS-micropillar. The optical excitation power incident on the SPS-micropillar can be controlled via the continuous injection current or voltage pulse height applied to the microlaser. Monolithic integration allows for nanometer precision in the distance between the microlaser and the SPS-micropillar $(15 \mu \mathrm{m})$, resulting in controlled and stable coupling of the structures. The p-contact on the SPS-micropillar has an aperture for the vertical emission of single-photons in the DBR mode perpendicular to the emission plane of the WGM microlaser. The ring aperture provides lateral current injection and has no observable effect on the single-photon outcoupling efficiency for this pillar diameter. The total device area including pads for bond wires is $\sim 200 \mu \mathrm{m}^{2}$.

We investigated two devices each consisting of a microlaser and SPS-micropillar pair, referred to as device A and device B. The devices were characterized in a continuous-flow liquid-helium cryostat with a micro-photoluminescence $(\mu \mathrm{PL})$ spectroscopy setup using a microscope objective NA of 0.42 (detection spot $\sim 3 \mu \mathrm{m}$ in diameter) and having a spectral resolution of $100 \mu \mathrm{eV}$. Single photons are vertically emitted from a QD in the SPSmicropillar directly into the detection axis when the microlaser is electrically driven by a source-measurement unit, and the orthogonal microlaser emission is vertically scattered from nearby uncontacted walls, giving access to the laser mode optical characteristics. For timeresolved (TR) measurements, a voltage pulse generator with a variable repetition rate of up to $250 \mathrm{MHz}$ was used to trigger the microlaser with pulse amplitudes as high as $3 \mathrm{~V}$ and a variable DC offset. TR measurements were carried out on the microlaser and SPSmicropillar emission using an silicon-based single-photon counting module (SPCM) with a timing resolution of $250 \mathrm{ps}$ and time-correlating electronics triggered by the pulse generator. For measurements of the photon autocorrelation function $g^{(2)}(\tau)$, two SPCMs together with a 50/50 fiber-beamsplitter were used with the timing electronics in an Hanbury-Brown and Twiss (HBT) configuration. A temperature-controller and heater were used to monitor and 
control the temperature of the devices from $5-40 \mathrm{~K}$.

\section{Results}

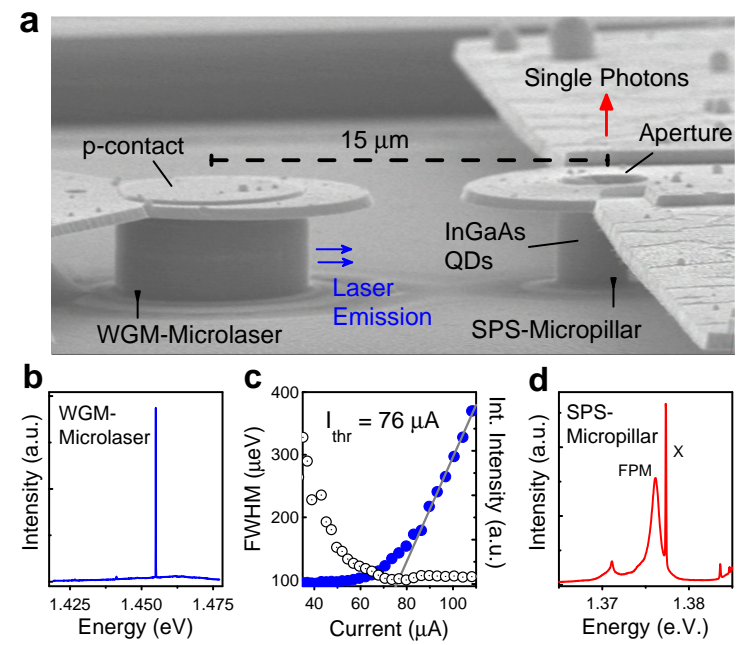

Figure 1: Sample layout and optical characterization of device A at $30 \mathrm{~K}$. (a) An SEM image of a $6 \mu \mathrm{m}$-diameter whispering-gallery-mode (WGM) quantum dot (QD) microlaser monolithically integrated next to a $2 \mu \mathrm{m}$-diameter single-photon-source (SPS) micropillar. The QD is resonantly coupled to the fundamental pillar mode (FPM) and emits single photons vertically through the aperture in the contact, which have been created by the microlaser's horizontal excitation. (b) A spectrum of the microlaser emission above threshold showing single-mode lasing. (c) Integrated intensity (filled blue circles) and full-width at halfmaximum (FWHM, open black circles) of the laser mode emission plotted against injection current. The fitted laser-threshold current is $76 \mu \mathrm{A}$ (grey line). (d) A spectrum of the neighboring SPS-micropillar under strong microlaser excitation. The FPM and a bright, single-QD excitonic transition $(\mathrm{X})$ can be seen at $1.376 \mathrm{eV}$.

We first present an overview of the device layout and optical characteristics of device A at 30 K. Fig. 1(a) shows a scanning-electron microscope (SEM) image of the integrated SPS. A larger micropillar with a diameter of $6 \mu \mathrm{m}$ acts as a microlaser via WGM emission in the plane of the QD active layer, and this laser emission pumps a single QD in the cavity of the neighboring SPS-micropillar of smaller diameter $(2 \mu \mathrm{m})$. While the laser emission is isotropic in the horizontal plane, the single photons emitted from the excited QD are highly directional along the vertical axis through the aperture. As mentioned in the previous section, the SPSmicropillar and laser are embedded in the transparent polymer BCB $(n=1.54)$ under 
normal operation, but this polymer has been ashed away in this figure to better reveal the underlying micropillar and contact structure. The radial separation between the micropillar and the microlaser is $15 \mu \mathrm{m}$. The gold p-contact seen overhanging the micropillar serves two crucial purposes: a bias can be delivered to the contact which tunes the emission of the QD via the QCSE, and an aperture in the contact suppresses the unwanted stray laser emission in the desired single-photon emission. The SPS-micropillar bias-voltage is isolated from the laser bias-voltage by the separate p-contact layout. To optically characterize the laser emission, we focus the microscope objective on nearby uncontacted walls as seen in the background of Fig. 1(a), which scatter laser light into the vertical direction. Fig. 1(b) shows a typical spectrum of the single-mode microlaser driven above threshold at $130 \mu \mathrm{A}$. Fig. 1(c) shows the intensity input-output curve of the laser mode emission (blue solid circles) and the full-width at half-maximum (FWHM, black open circles) as collected with the spectroscopy setup and fitted using a Voigt function. Lasing is evidenced by the drop in FWHM to the setup resolution and the sudden increase in mode intensity. The laser threshold current is determined with a linear fit to the integrated intensity (grey line) and is found to be $76 \mu \mathrm{A}$. A spectrum of the SPS-micropillar as it is driven by the microlaser under strong excitation is shown in Fig. 1(d). Here we see the fundamental pillar mode (FPM) at $1.376 \mathrm{eV}$ and a bright excitonic transition $(\mathrm{X})$ slightly detuned to the blue side of the FPM. Here there is no external bias applied to the target SPS-micropillar. The FPM is spectrally detuned from the laser mode by $\sim 80 \mathrm{meV}$, and the laser provides wetting-layer excitation to $\mathrm{X}$.

We now turn our attention to the electro-optical tuning of an exciton X from the SPSmicropillar in device A, performed with a varying bias-voltage applied at the aperture contact, and compare this to the case of temperature tuning of the same $\mathrm{X}$ over the same energy range. Fig. 2(a) shows an intensity plot of $\mathrm{X}$ driven by the microlaser as the bias-voltage on the SPS-micropillar is varied from $0.5-1.3 \mathrm{~V}$. The temperature is held constant at $33 \mathrm{~K}$ and the microlaser is driven with a constant current of $90 \mu \mathrm{A}$. X is tuned over an absolute energy range of $1.1 \mathrm{meV}$ by the QCSE. The increasing bias-voltage blue-shifts the discrete energy 

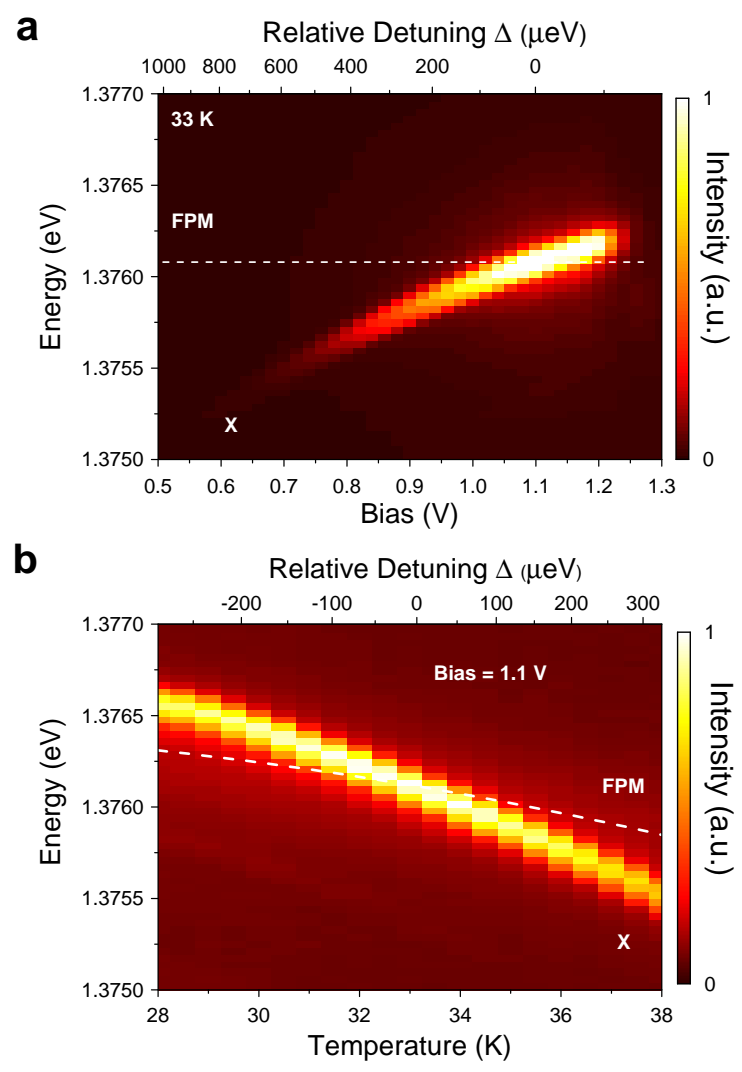

Figure 2: A single exciton (X) pumped by the integrated WGM microlaser in device A. (a) Electro-optical tuning of $\mathrm{X}$ through the FPM via an applied bias at $33 \mathrm{~K}$ and laser current of $90 \mu \mathrm{A}(\mathrm{b})$ Temperature-tuning of X through the FPM at a constant bias of $1.1 \mathrm{~V}$. 
levels of the QD but leaves the energy of the FPM unchanged, ${ }^{20}$ and X is electro-optically tuned into resonance with the FPM at a bias of $1.1 \mathrm{~V}$. Purcell enhancement as well as a gradual increase in intensity due to flattening of the built-in potential, which reduces tunneling of the charge carriers out of the QD, can both be seen. ${ }^{20}$ The intensity of X abruptly quenches at $\sim 1.25 \mathrm{~V}$, most likely due to a nearby trap becoming resonant with $\mathrm{X}$ providing a faster, non-radiative recombination channel. For comparison, Fig. 2(b) shows X at a constant bias of $1.1 \mathrm{~V}$ being temperature-tuned through resonance with the FPM under the same excitation conditions and over the same absolute energy range of $1.1 \mathrm{meV}$. The relative detuning between $\mathrm{X}$ and the FPM emission energy $\Delta=E_{\mathrm{FPM}}-E_{\mathrm{X}}$ is shown in the top axes of the intensity plots for both methods of tuning, and a crucial difference can be seen: the maximum relative detuning $\Delta$ for the electro-optical case is $820 \mu \mathrm{eV}$. In contrast, by temperature tuning $\mathrm{X}$ over the same $1.1 \mathrm{meV}$ absolute energy range corresponding to a temperature increase of $10 \mathrm{~K}$, the relative detuning between $\mathrm{X}$ and the FPM is $320 \mu \mathrm{eV}$. This is because heating simultaneously red-shifts $\mathrm{X}$ and the FPM, making the relative tunability between the two much smaller than in the case of electro-optical tuning. This becomes important when one considers that the FWHM of the FPM is a factor $\sim 10$ greater than that of X, meaning a larger tunability is useful if Purcell-enhanced emission is to be achieved. In addition to the points raised in the introduction about scalability, this is another advantage of an applied electric field as a tuning mechanism.

The electro-optically tuned SPS-micropillar emission properties of device A are examined in closer detail in Fig. 3. Two spectra showing $\mathrm{X}$ in and out of resonance with the FPM are shown in Fig. 3(a). The detuning between $\mathrm{X}$ and the FPM is out of the range shown in the color plot of Fig. 2(b), but the excitation conditions and bias are the same. The microlaser is operated at $90 \mu \mathrm{A}$ in both cases and the resonance temperature is $33 \mathrm{~K}$. The FWHM of $\mathrm{X}$ in resonance is $140 \mu \mathrm{eV}$, which is much larger than typical linewidths for InGaAs QDs. ${ }^{25}$ This is because the fine-structure splitting between horizontal and vertical polarization channels for X was found to be $40 \mu \mathrm{eV}$ (not shown) and as the setup resolution 

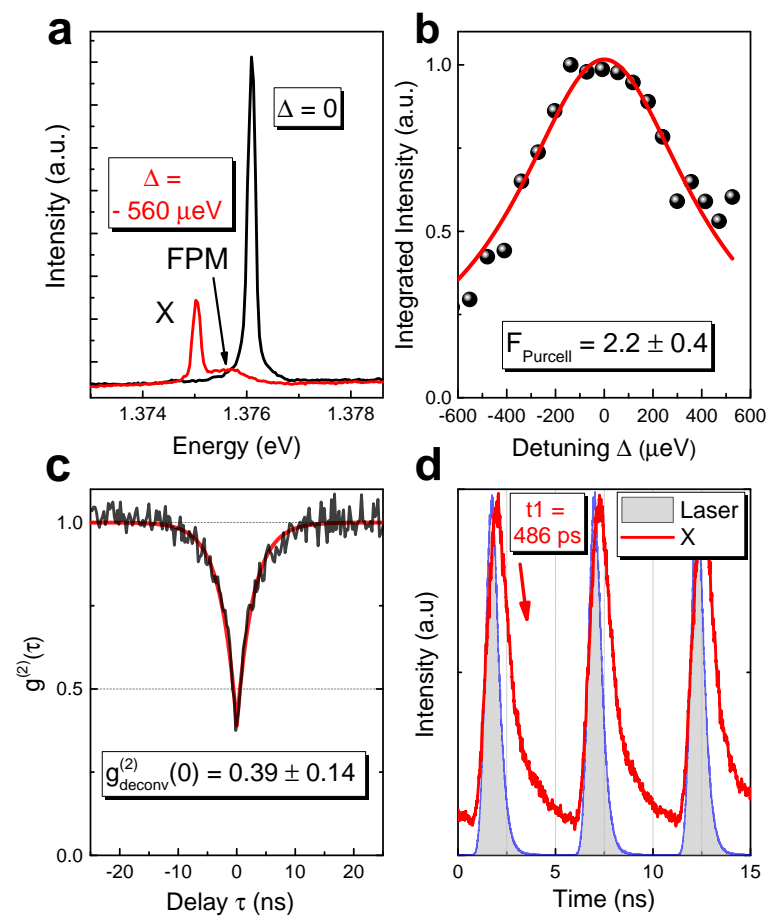

Figure 3: Emission properties of the electro-optically tuned $\mathrm{X}$ in device $\mathrm{A}$ at $1.1 \mathrm{~V}$ bias. (a) Two spectra showing X and the FPM in and out of resonance at $33 \mathrm{~K}$ and $42 \mathrm{~K}$, respectively. (b) The fitted Purcell factor for the temperature tuning of $\mathrm{X}$ is $2.2 \pm 0.4$. (c) Non-classical light emission of $\mathrm{X}$ at $33 \mathrm{~K}$ under constant laser excitation is verified by the fitted value of $g^{(2)}(0)=0.41 \pm 0.14$ and the deconvoluted value of $g_{\text {deconv }}^{(2)}(0)=0.39 \pm 0.14$. (d) Timeresolved measurements show the microlaser pulses (grey filled curves) have an FWHM of $\sim 780 \mathrm{ps}$ and drive the exciton $\mathrm{X}$ (red curve) which has a decay time of $(0,49 \pm 0.04) \mathrm{ns}$. 
is $100 \mu \mathrm{eV}$, the individual transitions themselves are resolution-limited. The emission of $\mathrm{X}$ is enhanced by the Purcell effect when the FPM and X spectrally overlap. The integrated intensity of $\mathrm{X}$ over a temperature range of $18 \mathrm{~K}$ was fitted in Fig. 3(b) using the fit function in Ref. ${ }^{26}$ From this fit, a Purcell factor $F_{P}=2.2 \pm 0.4$ is extracted. To verify the non-classical nature of the cavity-resonant emission of $\mathrm{X}$ under constant microlaser excitation, the light was analyzed with the HBT-setup as shown in Fig. 3(c). The characteristic dip at zerodelay in the autocorrelation function which falls below 0.5 is proof that $\mathrm{X}$ is a single-photon emitter driven by an integrated microlaser, with a raw fitted value of $g^{(2)}(0)=0.41 \pm 0.14$. A deconvolution of the signal accounting for the finite time-resolution of the SPCMs yields $g_{\text {deconv }}^{(2)}(0)=0.39 \pm 0.14$. Here, the non-ideal QD-lifetime is explained by the illumination of the cavity mode by non-resonant spectator QDs and the associated uncorrelated background emission. The time-resolved measurement of the lifetime of $\mathrm{X}$ in resonance with the FPM under pulsed microlaser excitation at $190 \mathrm{MHz}$ is shown in Fig. 3(d). The lifetime extracted from an exponential fit was found to be $(486 \pm 42) \mathrm{ps}$, which is about a factor 2.5 smaller than the typical value of $1.2 \mathrm{~ns}$ for these quantum dots in the absence of the upper DBR. This agrees well with the fitted Purcell factor $\left(F_{P}=2.2 \pm 0.4\right)$ of Fig. 3(b). This decay time is however clearly shorter than that suggested by the width of the anti-bunching dip in the continuous measurement of $g^{(2)}(\tau)$ shown in fig. 3(c), which indicates that the width of the dip in fig. 3(c) is limited by slow filling processes of the QD at a timescale longer than the QD lifetime. The laser pulses themselves, represented by the filled grey curves coinciding with X, have an FWHM of $780 \mathrm{ps}$ and a decay time of $343 \mathrm{ps}$. This is promising with respect to on-demand resonant excitation of these QDs, as the laser pulse decays faster than the QD. Furthermore, Fabry-Pérot spectrometer measurements (not shown) reveal FWHMs $<2 \mu \mathrm{eV}$ typical for these microlasers at currents of $\sim 100 \mu \mathrm{A}$, which is on the same order of magnitude as the homogeneous linewidth, and an order of magnitude smaller than the inhomogeneously-broadened linewidth (due to spectral diffusion) of the single-QD excitonic transitions. 

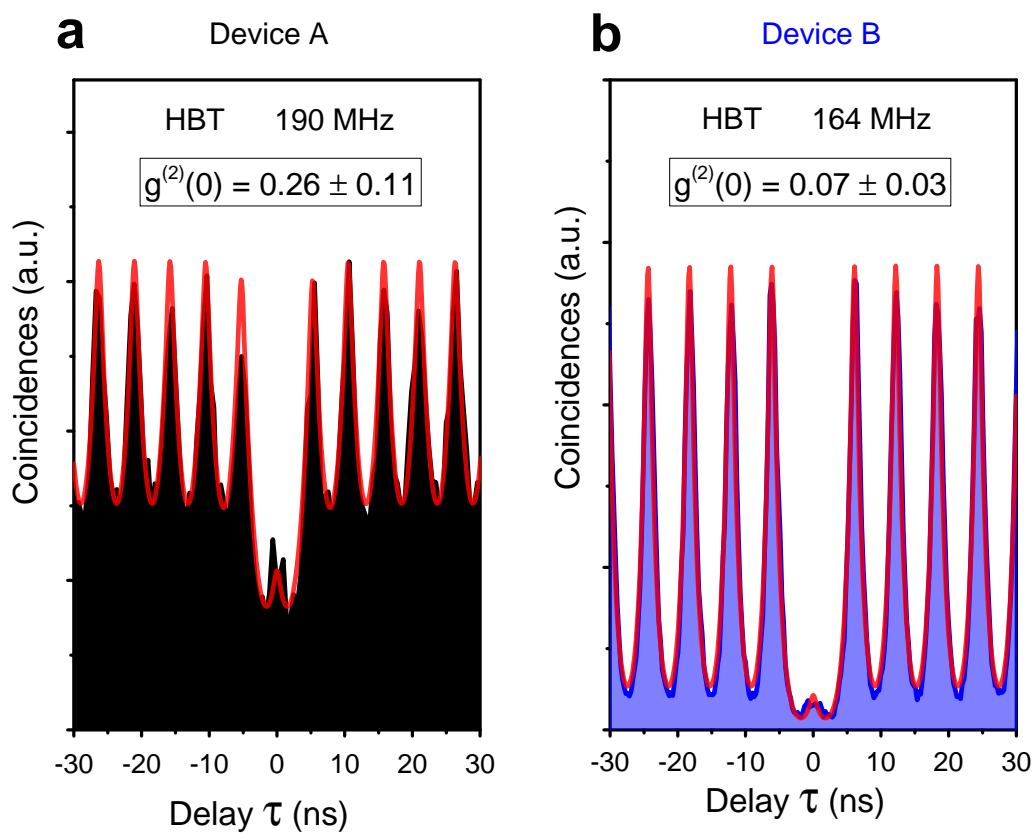

Figure 4: Pulsed, second-order correlation functions $g^{(2)}(\tau)$ measured in the Hanbury-Brown Twiss (HBT) configurations. (a) The electro-optically tuned $\mathrm{X}$ of device $\mathrm{A}$, in resonance with the FPM under a bias of $1.1 \mathrm{~V}$, is pulsed by the WGM microlaser demonstrating singlephoton emission with $g^{(2)}(0)=0.26 \pm 0.11$ at $33 \mathrm{~K}$. (b) Device B demonstrating very pure single-photon emission with $g^{(2)}(0)=0.07 \pm 0.03$ in resonance with the FPM at $5 \mathrm{~K}$ and with no bias-voltage. 
Fig. 4(a) shows a measurement of $g^{(2)}(\tau)$ on the $\mathrm{X}$ of device A, again with the $1.1 \mathrm{~V}$ bias and in resonance with the FPM at $33 \mathrm{~K}$, but now under pulsed microlaser excitation. The pulse repetition rate was $190 \mathrm{MHz}$. A fit to the peak areas gives a value of $g^{(2)}(0)=$ $0.26 \pm 0.11$, clearly below 0.5 and representative of a truly single-photon emitter. One reason for the improvement of the measured single-photon purity under pulsed excitation compared to constant excitation is because in the former case, the threshold of the laser is reduced, and this in turn reduces the background emission of the laser relative to the lasing mode intensity. The gain spectrum of the microlaser is given by the homogeneously broadened QD-distribution, which has a Gaussian FWHM of $30 \mathrm{meV}$. Therefore the tail of the gain spectrum which overlaps with the FPM of the SPS-micropillar is reduced under pulsed excitation, and the effect of stray laser background light in the measurement of $g^{(2)}(\tau)$ is reduced. In Fig. 4(b) we present $g^{(2)}(\tau)$ data of another SPS-micropillar driven by an integrated, low-threshold, single-mode microlaser (device B, on the same chip). This device demonstrates better performance in terms of a greatly reduced $g^{(2)}(0)=0.07 \pm 0.03$ because of reduced impact of uncorrelated emission of spectator QDs, but this excitonic transition was not tunable via an electric field as device A was, most likely due to the weak confinement of charge carriers typical in these quantum dots. The excitonic transition $\mathrm{X}$ for device $\mathrm{B}$ was in resonance with the FPM of a $2.7 \mu \mathrm{m}$-diameter micropillar at $5 \mathrm{~K}$, with no bias applied at the SPS-micropillar contact.

\section{Conclusion}

The results presented in this letter demonstrate the operation of QD-based, electrically triggered single-photon sources that show high purity $\left(g^{(2)}(0)\right.$ as low as $\left.0.07 \pm 0.03\right)$ and are driven by low-threshold microlasers integrated on the same chip. Electro-optical tuning of a single-QD exciton via an applied bias is demonstrated over a $1.1 \mathrm{meV}$ energy range. The advantages of this tuning mechanism in comparison to temperature tuning are indicated 
by the larger relative detuning between the cavity mode and the exciton, as well as being a tuning mechanism that is localized to the dimensions of the micropillar. The cavity-enhanced emission in resonance with the fundamental mode of the micropillar reduces the lifetime of the emitter by more than half with a Purcell factor of $2.2 \pm 0.4$. The excitation geometry is such that the laser emission and single-photon emission are orthogonal to one another, and the microlaser has a narrow linewidth and short pulse duration. These features, together with the results presented in this letter, demonstrate the suitability of this technology for future on-chip resonance-fluorescence experiments. Further optimization will be required to close the $80 \mathrm{meV}$ gap between the single-QD emission at the pillar mode and the laser mode, which will be technologically challenging as the QD-distribution must simultaneously be sufficient for lasing in the microlaser and detection at the single-QD level in the micropillar. The confinement potential must also be improved to allow for large tuning ranges via the applied electric field and to increase the yield of such micropillars. If these demands can be met, this concept may enable single, indistinguishable-photon turnstile devices for the betterment of emerging quantum technologies.

\section{Acknowledgement}

The authors thank M. Emmerling for expert sample preparation. This work was supported by the German Research Foundation (DFG) under Grant Nos. RE2974/9 - 1 and SCHN1376/1 - 1 and the German Federal Ministry of Education and Research (BMBF) for support through the VIP-project QSOURCE (Grant No. 03V0630).

\section{References}

(1) Chunnilall, C. J.; Degiovanni, I. P.; Kück, S.; Müller, I.; Sinclair, A. G. Metrology of Single-photon Sources and Detectors: a Review. Opt. Eng. 2014, 53, 081910. 
(2) Aharonovich, I.; Englund, D.; Toth, M. Solid-state Single-photon Emitters. Nat. Photon. 2016, 10, 631-641.

(3) Bentham, C.; Hallett, D.; Prtljaga, N.; Royall, B.; Vaitiekus, D.; Coles, R. J.; Clarke, E.; Fox, A. M.; Skolnick, M. S.; Itskevich, I. E.; et al., Single-photon Electroluminescence for On-chip Quantum Networks. Appl. Phys. Lett. 2016, 109, 161101.

(4) Farahani, J. N.; Pohl, D. W.; Eisler, H.-J.; Hecht, B. Single Quantum Dot Coupled to a Scanning Optical Antenna: A Tunable Superemitter. Phys. Rev. Lett. 2005, 95.

(5) Lee, K. G.; Chen, X. W.; Eghlidi, H.; Kukura, P.; Lettow, R.; Renn, A.; Sandoghdar, V.; Götzinger, S. A Planar Dielectric Antenna for Directional Single-photon Emission and Near-unity Collection Efficiency. Nat. Photon. 2011, 5, 166-169.

(6) Claudon, J.; Bleuse, J.; Malik, N. S.; Bazin, M.; Jaffrennou, P.; Gregersen, N.; Sauvan, C.; Lalanne, P.; Gérard, J.-M. A Highly Efficient Single-photon Source based on a Quantum Dot in a Photonic Nanowire. Nat. Photon. 2010, 4, 174-177.

(7) Gschrey, M.; Thoma, A.; Schnauber, P.; Seifried, M.; Schmidt, R.; Wohlfeil, B.; Krüger, L.; Schulze, J. H.; Heindel, T.; Burger, S.; Schmidt, F.; Strittmatter, A.; Rodt, S.; Reitzenstein, S. Highly Indistinguishable Photons from Deterministic Quantum-dot Microlenses utilizing Three-dimensional in situ Electron-beam Lithography. Nat. Commun. 2015, 6, 7662.

(8) Heindel, T.; Schneider, C.; Lermer, M.; Kwon, S. H.; Braun, T.; Reitzenstein, S.; Höfling, S.; Kamp, M.; Forchel, A. Electrically Driven Quantum Dot-micropillar Single Photon Source with 34\% Overall Efficiency. Appl. Phys. Lett. 2010, 96, 011107.

(9) Ding, X.; He, Y.; Duan, Z.-C.; Gregersen, N.; Chen, M.-C.; Unsleber, S.; Maier, S.; Schneider, C.; Kamp, M.; Höfling, S.; et al., On-Demand Single Photons with High Extraction Efficiency and Near-Unity Indistinguishability from a Resonantly Driven Quantum Dot in a Micropillar. Phys. Rev. Lett. 2016, 116. 
(10) Dietrich, C. P.; Fiore, A.; Thompson, M. G.; Kamp, M.; Höfling, S. GaAs Integrated Quantum Photonics: Towards Compact and Multi-functional Quantum Photonic Integrated Circuits. Laser Photon. Rev. 2016, 10, 870-894.

(11) Santori, C.; Fattal, D.; Vučković, J.; Solomon, G. S.; Yamamoto, Y. Indistinguishable Photons from a Single-photon Device. Nature 2002, 419, 594-597.

(12) Somaschi, N. et al. Near-optimal Single-photon Sources in the Solid State. Nat. Photon. 2016, 10, 340-345.

(13) Unsleber, S.; He, Y.-M.; Gerhardt, S.; Maier, S.; Lu, C.-Y.; Pan, J.-W.; Gregersen, N.; Kamp, M.; Schneider, C.; Höfling, S. Highly Indistinguishable On-demand Resonance Fluorescence Photons from a Deterministic Quantum Dot Micropillar Device with 74\% Extraction Efficiency. Opt. Express 2016, 24, 8539.

(14) Stock, E.; Albert, F.; Hopfmann, C.; Lermer, M.; Schneider, C.; Höfling, S.; Forchel, A.; Kamp, M.; Reitzenstein, S. On-Chip Quantum Optics with Quantum Dot Microcavities. Adv. Mater. 2012,

(15) Kremer, P. E.; Dada, A. C.; Kumar, P.; Ma, Y.; Kumar, S.; Clarke, E.; Gerardot, B. D. Strain-tunable Quantum dot Embedded in a Nanowire Antenna. Phys. Rev. B 2014, 90.

(16) Robinson, J. W.; Rice, J. H.; Lee, K. H.; Na, J. H.; Taylor, R. A.; Hasko, D. G.; Oliver, R. A.; Kappers, M. J.; Humphreys, C. J.; Briggs, G. A. D. Quantum-confined Stark Effect in a Single InGaN Quantum Dot under a Lateral Electric Field. Appl. Phys. Lett. 2005, 86, 213103.

(17) Zhang, Y.; Chen, Y.; Mietschke, M.; Zhang, L.; Yuan, F.; Abel, S.; Hühne, R.; Nielsch, K.; Fompeyrine, J.; Ding, F.; et al., Monolithically Integrated Microelectromechanical Systems for On-Chip Strain Engineering of Quantum Dots. Nano Lett. 2016, $16,5785-5791$. 
(18) Findeis, F.; Baier, M.; Beham, E.; Zrenner, A.; Abstreiter, G. Photocurrent and Photoluminescence of a Self-assembled Quantum Dot in Electric Fields. Appl. Phys. Lett. 2001, 78, 2958-2960.

(19) Beetz, J.; Kistner, C.; Lermer, M.; Schneider, C.; Reitzenstein, S.; Höfling, S.; Kamp, M.; Forchel, A. In-plane Manipulation of Quantum Dots in High Quality Laterally Contacted Micropillar Cavities. Appl. Phys. Lett. 2011, 98, 191111.

(20) Kistner, C.; Heindel, T.; Schneider, C.; Rahimi-Iman, A.; Reitzenstein, S.; Höfling, S.; Forchel, A. Demonstration of Strong Coupling via Electro-optical Tuning in Highquality QD-micropillar Systems. Opt. Express 2008, 16, 15006-15012.

(21) Laucht, A.; Hofbauer, F.; Hauke, N.; Angele, J.; Stobbe, S.; Kaniber, M.; Bohm, G.; Lodahl, P.; Amann, M.-C.; Finley, J. J. Electrical Control of Spontaneous Emission and Strong Coupling for a Single Quantum Dot. New J. Phys. 2009, 11, 023034 (11pp).

(22) Bennett, A. J.; Patel, R. B.; Skiba-Szymanska, J.; Nicoll, C. A.; Farrer, I.; Ritchie, D. A.; Shields, A. J. Giant Stark Effect in the Emission of Single Semiconductor Quantum Dots. Appl. Phys. Lett. 2010, 97, 031104.

(23) Munnelly, P.; Heindel, T.; Karow, M. M.; Höfling, S.; Kamp, M.; Schneider, C.; Reitzenstein, S. A Pulsed Nonclassical Light Source Driven by an Integrated Electrically Triggered Quantum Dot Microlaser. IEEE J. Sel. Top. Quantum Electron. 2015, 21, $681-689$.

(24) Schlehahn, A.; Thoma, A.; Munnelly, P.; Kamp, M.; Höfling, S.; Heindel, T.; Schneider, C.; Reitzenstein, S. An Electrically Driven Cavity-enhanced Source of Indistinguishable Photons with 61\% Overall Efficiency. APL Photonics 2016, 1.

(25) Bayer, M.; Ortner, G.; Stern, O.; Kuther, A.; Gorbunov, A. A.; Forchel, A.; Hawrylak, P.; Fafard, S.; Hinzer, K.; Reinecke, T. L.; Walck, S. N.; Reithmaier, J. P.; 
Klopf, F.; Schäfer, F. Fine Structure of Neutral and Charged Excitons in Self-assembled In(Ga)As/(Al)GaAs Quantum Dots. Phys. Rev. B 2002, 65, 195315.

(26) Munsch, M.; Mosset, A.; Auffèves, A.; Seidelin, S.; Poizat, J. P.; Gérard, J.-M.; Lemaître, A.; Sagnes, I.; Senellart, P. Continuous-wave versus Time-resolved Measurements of Purcell Factors for Quantum Dots in Semiconductor Microcavities. Phys. Rev. B 2009, 80, 115312 . 
Graphical TOC Entry

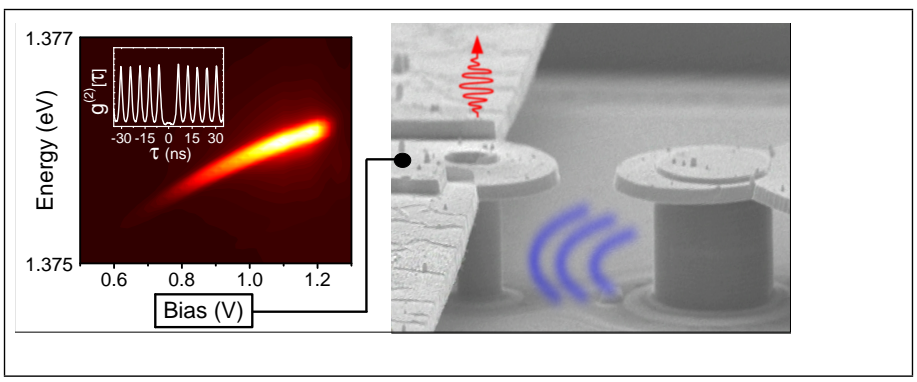

